\title{
The use of e-cigarettes and its risk of respiratory diseases
}

\author{
Hamed Saeed Al-Ghamdi ${ }^{1 *}$, Nader Ghazi Althagafi ${ }^{2}$, Meshal Jamal Alshawaf ${ }^{3}$, \\ Osama Mohammed Allathiqani ${ }^{4}$, Reem Abdulsalam Almaflehi ${ }^{5}$, \\ Fahad Abdulrahman Almuzaini ${ }^{6}$, Munirah Salem Almohammadi ${ }^{7}$, Mazen Ibrahim Alzahrani ${ }^{8}$, \\ Fatimah Mohammed Alshakhs, ${ }^{9}$ Asala Mamdouh Wafa ${ }^{5}$, Saleh Mubarak Alajmi ${ }^{10}$
}

\author{
${ }^{1}$ Department of Internal Medicine, King Abdulaziz Hospital, Jeddah, Saudi Arabia \\ ${ }^{2}$ College of Medicine, Medical University of Lodz, Lodz, Poland \\ ${ }^{3}$ College of Medicine, King Faisal University, Al Ahsa, Saudi Arabia \\ ${ }^{4}$ College of Medicine, Taibah University, Medina, Saudi Arabia \\ ${ }^{5}$ College of Medicine, Batterjee Medical College, Jeddah, Saudi Arabia \\ ${ }^{6}$ Emergency Medical Services, Al Bukayriyah General Hospital, Al Bukayriyah, Saudi Arabia \\ ${ }^{7}$ Department of Internal Medicine, King Salman Medical City, Medina, Saudi Arabia \\ ${ }^{8}$ College of Medicine, King Abdulaziz University, Rabigh, Saudi Arabia \\ ${ }^{9}$ College of Medicine, Baha University, Baha, Saudi Arabia \\ ${ }^{10}$ College of Medicine, Jordan University of Science and Technology, Irbid, Jordan
}

Received: 01 October 2021

Accepted: 16 October 2021

\author{
*Correspondence: \\ Dr. Hamed Saeed Al-Ghamdi, \\ E-mail: h.s.alghamdi@hotmail.com
}

Copyright: ( ) the author(s), publisher and licensee Medip Academy. This is an open-access article distributed under the terms of the Creative Commons Attribution Non-Commercial License, which permits unrestricted non-commercial use, distribution, and reproduction in any medium, provided the original work is properly cited.

\begin{abstract}
There are increasing concerns about the impact of using e-cigarettes on the overall health and respiratory complications of users. These modalities were proposed to potentially reduce the harmful events that are associated with cigarette smoking. Nevertheless, the acute and chronic effects secondary to the exposure and use of this modality are still controversial. However, it has been demonstrated that using the modality is associated with remarkable damage to lung injury because it releases significant amounts of reactive oxygen species, furans, volatile carbonyls, furans and metals (chromium, lead, and nickel). We have discussed the impact of e-cigarette using and exposure to its aerosol and the development and progression of the different respiratory diseases at the different clinical and pathological levels. Asthma, chronic bronchitis were the most significant lung conditions, and evidence indicated that exacerbation of symptoms was reported in some patients. Furthermore, acute exposure was also associated with the development of respiratory-related symptoms. Accordingly, studies indicated severe inflammatory reactions and significant lung injury were observed among e-cigarette users concerning vaping or exposure. Therefore, further preventive approaches are required by healthcare authorities to increase public awareness of the different hazards of using these modalities.
\end{abstract}

Keywords: E-cigarette, Smoking, Damage, Respiratory diseases, Lung toxicity

\section{INTRODUCTION}

The role of the respiratory system in human physiology is critical as the lungs transmit the total cardiac output via around $2000 \mathrm{~km}$ of capillaries. The estimated surface area of the alveoli is $70 \mathrm{~m}^{2}$ and the heat beats through the vast space in these alveoli performing the process of gas exchange. Furthermore, it is known that the lungs are mainly at high risk of catching a disease because they are usually exposed to the different inflammatory and infectious diseases with every breath taken. However, the inspired air is humidified and cleaned as a result of the underlying structure of the respiratory system and the 
presence of acquired and innate immunity, which keeps the delicate structure of the lungs and alveoli protected. ${ }^{1}$

There are increasing concerns about the impact of using e-cigarettes on the overall health and respiratory complications of users. These modalities were proposed to potentially reduce the harmful events that are associated with cigarette smoking. ${ }^{2}$ Nevertheless, the acute and chronic effects secondary to the exposure and use of this modality are still controversial. However, it has been demonstrated that using the modality is associated with remarkable damage to lung injury because it releases significant amounts of reactive oxygen species, furans, volatile carbonyls, furans and metals (chromium, lead and nickel). ${ }^{3}$ In the present literature review, we aimed to provide evidence regarding the impact of e-cigarette use and the development and progression of respiratory diseases in these users.

\section{METHODS}

This literature review was based on an extensive literature search in the Medline, Cochrane and EMBASE databases on which was performed 26 September 2021 using the medical subject headings (MeSH) or a combination of all possible related terms, according to the database. To avoid missing poetential studies, a further manual search for papers was done through Google Scholar, while the reference lists of the initially included papers. Studies discussing the impact of e-cigarette use on the progression of respiratory diseases were screened for useful information, with no limitations posed on date, language, age of participants or publication type.

\section{DISCUSSION}

The rates of cigarette smoking were significant in the late 19th and early 20th centuries following the huge advances in mass production. However, the significance of the impact of cigarette smoking was not concerned until the 1930s, when evidence showed that the prevalence of lung cancer and associated different surgical procedures including pneumonectomy, in addition to the remarkable increase in the mortality rates owing to thoracic cancers. ${ }^{4,5}$ Following this, the US surgeon general's report that was published in 1964 demonstrated that lung cancer was significantly associated with cigarette smoking. Nevertheless, the cigarette smoking industry was not burdened until four decades later. Accordingly, the progression of the education about the harmful effects of cigarette smoking and its correlation with the different respiratory diseases took much time before making a significant impact on the industrial process. Therefore, the current healthcare authorities should exert further efforts about all the available tobacco products to furtherly investigate the impact of these modalities on the health status of smokers. In e-cigarettes, nicotine, propylene glycol and vegetable glycerin, in addition to the different flavoring agents were heated and aerosolized using a metal resistance coil. Many changes were introduced to these modalities to enhance their functions and make them more advantageous. The main aim of these modalities was to make the user better control of the administered nicotine concentration, e-liquid composition and vaping of e-liquids.

Many previous investigations have indicated the association between the increased risk of different respiratory diseases and pulmonary lesions with the use of e-cigarettes. A previous investigation by Cho et al also reported that as a result of asthma-induced frequent use of e-cigarettes, the affected patients had a more frequent absence from schools. ${ }^{6}$ This was also indicated in high school students that exhibited asthma-like symptoms secondary to the use of e-cigarettes. ${ }^{7,8}$ It was worth mentioning that the previous investigation by Cho et al demonstrated that there was no significant association between asthma and e-cigarette use among smokers. Nonetheless, the correlation was significant among neversmokers. ${ }^{6}$ This had been further indicated in another investigation by Wang et al that demonstrated that there was a significant correlation between respiratory symptoms and e-cigarette use among never-smokers. ${ }^{9}$ In the United States, an investigation by Choi et al demonstrated that 30 day use of e-cigarette was more significantly correlated with the development of respiratory symptoms than lifetime use. ${ }^{10}$ This had been furtherly indicated in other investigations that indicated the strong association between asthma development and e-cigarette use. ${ }^{11,12}$ McConnell et al also conducted a study in California and found that chronic bronchitis was significantly associated with e-cigarette use and the association was dose-dependent. ${ }^{13}$ Chronic obstructive pulmonary disease (COPD) was also significantly associated with the prolonged use of e-cigarettes. In the United States, evidence showed that the use of these modalities, especially among dual smokers was significantly associated with a diagnosis of COPD. ${ }^{14-16}$ Wills et al also demonstrated that the association of COPD and e-cigarette use was more significant among non-smokers than the included individuals that smoked. ${ }^{17}$ Furthermore, among healthy individuals, evidence indicated that exposure to e-cigarettes aerosol was significantly associated with the development of respiratory symptoms. Besides, this had been associated with increased symptoms of asthma, COPD, cystic fibrosis, in addition to the altered host defense status and respiratory physiology. Studies also demonstrated that phlegm and cough have been significantly associated with the use of e-cigarettes among adolescents.

Different investigations have also assessed the association between the use of e-cigarettes and respiratory diseases through spirometry and evaluation of respiratory functions. Most of these studies have focused on the airway changes that occur immediately after vaping to assess the acute inflammatory changes. The findings regarding the potential association between respiratory diseases and the use of e-cigarettes among these studies 
were controversial. It should be noted that it had been indicated that acute airway obstruction can significantly develop after exposure to e-cigarettes in patients suffering from pre-existing airway conditions. ${ }^{18,19}$ On the other hand, only a few spirometric investigations have assessed the association on a long-term basis. A previous investigation compared e-cigarette users (and excluded current and previous smokers) with controls and found that the respiratory functions were significantly reduced among e-cigarette users. ${ }^{20}$ However, it should be noted that the authors of this investigation indicated that assessment of the included patients was done 1 hour after abstinence. Therefore, the worsened functions among individuals using e-cigarettes might be attributable to the potential acute spasm secondary to using e-cigarettes. Other investigations also demonstrated that no significant changes or improvements in their respiratory functions were noticed after to using e-cigarettes. ${ }^{21,22}$ Besides, it should be noted that normal respiratory function tests on spirometry do not necessarily mean that using e-cigarettes was harmless. This can be elaborated by an example of patients that suffered from cystic fibrosis, in which the lungs of these patients progressively deteriorated due to this chronic lung disease, as younger patients usually have normal functions tests despite the underlying condition of their lungs. ${ }^{23}$ Similarly, abnormal functions tests secondary to e-cigarettes use can then be detected years after exposure and use of these modalities. However, it should be noted that a distal injury of the lung tissues was still inevitable and can be evaluated by other approaches. ${ }^{24}$ For instance, evidence indicated the ability of imaging modalities on detecting remarkable pathological changes that might include alveolar destruction, similar to the pathology of COPD secondary to smoking, although the corresponding changes to the respiratory functions were marked as relatively low. ${ }^{25}$

Previous investigations have also demonstrated the main mechanisms of the pathology of pulmonary diseases that were induced by the use of e-cigarettes. Nitric oxide deficiency, increased oxidative stress and endothelial dysfunction was the main events that occured to these patients contributing to the pathology of the different conditions and pulmonary disease. ${ }^{26}$ Reduced clearance activities of the nasal and bronchial mucociliary have also been reported in previous investigations due to the exposure and use of e-cigarettes. Reduced nitric oxide and increased airway resistance among healthy adults were also significantly associated with the short-term exposure to e-cigarettes with and without nicotine within them. ${ }^{27,28}$ This had been furtherly indicated in other investigations that demonstrated that following the exposure of healthy individuals to e-cigarettes smoking, reduced pulmonary functions were significant among these individuals. Moreover, it should be noted that there was no sufficient evidence that can support the favorable outcomes of alternative use of e-cigarettes rather than the usual smoking as noticed with quitting. Lung toxicity and damage, in addition to bronchial and parenchymal inflammation, in addition to the impaired signaling of systemic inflammation were also reported in the literature secondary to e-cigarettes use and exposure. ${ }^{29-31}$

Evidence showed that mild changes can affect lung functions significantly because of the delicate structure of the lungs. It had been demonstrated that in patients with COPD, reduced respiratory functions were associated with mucin levels, which indicated the presence of significant harm and chronic bronchitis. Following exposure to e-cigarettes, previous investigations have indicated that the fractional excretion of nitric oxide had been estimated to significantly decrease. ${ }^{32}$ Further evidence indicated the elevated levels of neutrophils in the sputum of e-cigarette users including proteinase-3, neutrophil elastase and myeloperoxidase. This was also previously indicated to occur among tobacco smokers. ${ }^{33,34}$ Therefore, this indicated that the presence of these substances in e-cigarette users was a strong indicator of the presence of an underlying lung lesion. In the same context, a previous controlled investigation demonstrated that decreased expiratory gas flow and impaired gas exchange were significant after exposure to e-cigarettes aerosol, which was also associated with increased levels of lung-specific protein CC16. This showed that using ecigarettes can significantly cause lung damage in a physiologically acute pattern. ${ }^{35}$

Delayed recovery from and increased susceptibility to different respiratory infections have been associated with the use of e-cigarettes. ${ }^{36}$ Reduced cough sensitivity was reported in a previous investigation for participants that were exposed to e-cigarettes aerosol while being healthy and non-smokers. Previous animal investigations also reported that ciliary dysfunction can also be associated with these situations. Accordingly, increased rates of pneumonia might be observed among these patients as a result of the reduced ciliary clearance and reduced cough sensitivity following the exposure to e-cigarettes aerosol. ${ }^{37}$ Critical functions of the innate immunity of the affected patients might also be impaired in these situations. A previous investigation that compared nasal biopsies between smokers, non-smokers and e-cigarette users demonstrated that e-cigarette users had a significantly higher risk than the other two populations in terms of increased immunosuppression at a genetic level. ${ }^{38}$ Alveolar macrophages were also studied in a previous investigation that conducted an alveolar lavage for healthy non-smoking participants that were exposed to e-cigarettes aerosol. The authors demonstrated that following only 20 puffs by two hours from using ecigarettes, 60 genes were altered within the studied macrophages, including inflammation-related genes. Impaired neutrophil functions and phagocytosis were also reported to be potentially present among e-cigarette users.

In addition to the different human investigations, previous animal studies were also found in the literature, indicating the significant association between exposure to ecigarettes and the development of significant lung injury and subsequent pulmonary diseases. ${ }^{39,40}$ Different protein 
and cellular changes were reported in the literature secondary to the acute exposure to e-cigarettes aerosol including modulation of the functions of cytokines and mucins, in addition to the impaired autophagy. Furthermore, evidence also indicated that following chronic exposure, neutrophilia, emphysema and airway inflammation and remodeling were observed in the investigated animal models. ${ }^{41}$ Nicotine and non-nicotinedependent effects and increased sensitivity to methacholine were also reported by some studies. On the other hand, it should be noted that some previous investigations reported that vaping does not adversely impact the health status of the lungs. However, the results of these studies might be biased because they are industrial-based on similar findings were not reported by other investigations. ${ }^{42}$ Therefore, the current evidence still supported the aforementioned parameters regarding the adverse association between e-cigarette use and the health status of the users' lungs.

\section{CONCLUSION}

Asthma, chronic bronchitis were the most significant lung conditions and evidence indicates that exacerbation of symptoms was reported in some patients. Furthermore, acute exposure was also associated with the development of respiratory-related symptoms. Accordingly, studies indicated that severe inflammatory reactions and significant lung injury were observed among e-cigarette users in relation to vaping or exposure. Therefore, further preventive approaches are required by healthcare authorities to increase public awareness of the different hazards of using these modalities.

Funding: No funding sources Conflict of interest: None declared

Ethical approval: Not required

\section{REFERENCES}

1. Patwa A, Shah A. Anatomy and physiology of respiratory system relevant to anaesthesia. Indian J Anaesth. 2015;59(9):533-41.

2. Pierce JP, Chen R, Leas EC, White MM, Kealey S, Stone $\mathrm{MD}$, et al. Use of e-cigarettes and other tobacco products and progression to daily cigarette smoking. Pediatrics. 2021;147(2):2020025122.

3. National Academies of Sciences, Engineering, and Medicine, Health and Medicine Division, Board on Population Health and Public Health Practice, Committee on the Review of the Health Effects of Electronic Nicotine Delivery Systems, David L. Eaton, Leslie Y. Kwan, et al. Public health consequences of e-cigarettes. Washington: National Academies Press; 2018.

4. Courtney R. The Health Consequences of Smoking50 Years of Progress: A Report of the Surgeon General, 2014 Us Department of Health and Human Services Atlanta, GA: Department of Health and Human Services, Centers for Disease Control and
Prevention, National Center for Chronic Disease Prevention and Health Promotion, Office on Smoking and Health. Wiley Online Library; 2014: 1081.

5. Ochsner A, DeBakey M. Primary pulmonary malignancy: treatment by total pneumonectomy; analysis of 79 collected cases and presentation of 7 personal cases. Ochsner J. 1999;1(3):109.

6. Cho JH, Paik SY. Association between Electronic Cigarette Use and Asthma among High School Students in South Korea. PloS One. 2016;11(3):0151022.

7. Kim SY, Sim S, Choi HG. Active, passive, and electronic cigarette smoking is associated with asthma in adolescents. Sci Reps. 2017;7(1):17789.

8. Lee A, Lee SY, Lee KS. The use of heated tobacco products is associated with asthma, allergic rhinitis, and atopic dermatitis in Korean adolescents. Sci Reps. 2019;9(1):17699.

9. Wang MP, Ho SY, Leung LT, Lam TH. Electronic cigarette use and respiratory symptoms in Chinese adolescents in Hong Kong. JAMA Pediatr. 2016;170(1):89-91.

10. Choi K, Bernat D. E-cigarette use among Florida youth with and without asthma. Am J Prevent Med. 2016;51(4):446-53.

11. Fedele DA, Barnett TE, Dekevich D, Gibson-Young LM, Martinasek M, Jagger MA. Prevalence of and beliefs about electronic cigarettes and hookah among high school students with asthma. Ann Epidemiol. 2016;26(12):865-9.

12. Bayly JE, Bernat D, Porter L, Choi K. Second hand exposure to aerosols from electronic nicotine delivery systems and asthma exacerbations among youth with asthma. Chest. 2019;155(1):88-93.

13. McConnell R, Barrington-Trimis JL, Wang K, Urman R, Hong $\mathrm{H}$, Unger $\mathrm{J}$, et al. Electronic cigarette use and respiratory symptoms in adolescents. Am J Respir Crit Care Med. 2017;195(8):1043-9.

14. Wang JB, Olgin JE, Nah G, Vittinghoff E, Cataldo JK, Pletcher MJ, et al. Cigarette and e-cigarette dual use and risk of cardiopulmonary symptoms in the health eheart study. PloS One. 2018;13(7):0198681.

15. Li D, Sundar IK, McIntosh S, Ossip DJ, Goniewicz ML, O'Connor RJ, et al. Association of smoking and electronic cigarette use with wheezing and related respiratory symptoms in adults: cross-sectional results from the population assessment of tobacco and health (PATH) study, wave 2. Tobacco Control. 2020;29(2):140-7.

16. Osei AD, Mirbolouk M, Orimoloye OA, Dzaye O, Uddin SMI, Benjamin EJ, et al. Association between e-cigarette use and chronic obstructive pulmonary disease by smoking status: behavioral risk factor surveillance system 2016 and 2017. Am J Prev Med. 2020;58(3):336-42.

17. Wills TA, Pagano I, Williams RJ, Tam EK. Ecigarette use and respiratory disorder in an adult sample. Drug Alcohol Depend. 2019;194:363-70. 
18. Vardavas CI, Anagnostopoulos N, Kougias M, Evangelopoulou V, Connolly GN, Behrakis PK. Short-term pulmonary effects of using an electronic cigarette: impact on respiratory flow resistance, impedance, and exhaled nitric oxide. Chest. 2012;141(6):1400-6.

19. Flouris AD, Chorti MS, Poulianiti KP, Jamurtas AZ, Kostikas K, Tzatzarakis MN, et al. Acute impact of active and passive electronic cigarette smoking on serum cotinine and lung function. Inhal Toxicol. 2013;25(2):91-101.

20. Meo SA, Ansary MA, Barayan FR, Almusallam AS, Almehaid AM, Alarifi NS, et al. Electronic Cigarettes: Impact on Lung Function and Fractional Exhaled Nitric Oxide Among Healthy Adults. Am J Men Health. 2019;13(1):1557988318806073.

21. Cibella F, Campagna D, Caponnetto P, Amaradio MD, Caruso M, Russo C, et al. Lung function and respiratory symptoms in a randomized smoking cessation trial of electronic cigarettes. Clinic Sci. 2016;130(21):1929-37.

22. Veldheer S, Yingst J, Midya V, Hummer B, Lester C, Krebs N, et al. Pulmonary and other health effects of electronic cigarette use among adult smokers participating in a randomized controlled smoking reduction trial. Addict Behav. 2019;91:95101.

23. Aurora P, Stanojevic S, Wade A, Oliver C, Kozlowska W, Lum S, et al. Lung clearance index at 4 years predicts subsequent lung function in children with cystic fibrosis. Am J Respir Crit Care Med. 2011;183(6):752-8.

24. Woodruff PG, Barr RG, Bleecker E, Christenson SA, Couper D, Curtis JL, et al. Clinical significance of symptoms in smokers with preserved pulmonary function. N Engl J Med. 2016;374(19):1811-21.

25. Coxson HO, Leipsic J, Parraga G, Sin DD. Using pulmonary imaging to move chronic obstructive pulmonary disease beyond FEV1. Am J Respir Crit Care Med. 2014;190(2):135-44.

26. Malerba M, Radaeli A, Olivini A, Damiani G, Ragnoli B, Montuschi P, et al. Exhaled nitric oxide as a biomarker in COPD and related comorbidities. Bio Med Res Int. 2014;2014:271918.

27. Marini S, Buonanno G, Stabile L, Ficco G. Shortterm effects of electronic and tobacco cigarettes on exhaled nitric oxide. Toxicol App Pharmacol. 2014;278(1):9-15.

28. Muley T, Wiebel M, Schulz V, Ebert W. Elastinolytic activity of alveolar macrophages in smoking-associated pulmonary emphysema. Clinic Investigat. 1994;72(4):269-76.

29. Harris JO, Olsen GN, Castle JR, Maloney AS. Comparison of proteolytic enzyme activity in pulmonary alveolar macrophages and blood leukocytes in smokers and nonsmokers. Am Rev Respir Dis. 1975;111(5):579-86.
30. Takahashi H, Ishidoh K, Muno D, Ohwada A, Nukiwa T, Kominami E, et al. Cathepsin L activity is increased in alveolar macrophages and bronchoalveolar lavage fluid of smokers. Am Rev Respir Dis. 1993;147:1562-8.

31. Churg A, Cosio M, Wright JL. Mechanisms of cigarette smoke-induced COPD: insights from animal models. Am J Physiol Lung Cell Mol Physiol. 2008;294(4):612-31.

32. Sohal SS, Reid D, Soltani A, Ward C, Weston S, Muller HK, et al. Reticular basement membrane fragmentation and potential epithelial mesenchymal transition is exaggerated in the airways of smokers with chronic obstructive pulmonary disease. Respirology. 2010;15(6):930-8.

33. Crystal RG. Alpha 1-antitrypsin deficiency, emphysema, and liver disease. Genetic basis and strategies for therapy. $\mathbf{J}$ Clinic Invest. 1990;85(5):1343-52.

34. Stick SM, Brennan S, Murray C, Douglas T, Ungern-Sternberg BSV, Garratt LW, et al. Bronchiectasis in infants and preschool children diagnosed with cystic fibrosis after newborn screening. J Pediatr. 2009;155(5):623-8.

35. Mason SD, Joyce JA. Proteolytic networks in cancer. Trend Cell Biol. 2011;21(4):228-37.

36. Moazed F, Calfee CS. Environmental risk factors for acute respiratory distress syndrome. Clinic Chest Med. 2014;35(4):625-37.

37. Viswam D, Trotter S, Burge PS, Walters GI. Respiratory failure caused by lipoid pneumonia from vaping e-cigarettes. BMJ Case Rep. 2018;2018.

38. Agustin M, Yamamoto M, Cabrera F, Eusebio R. Diffuse alveolar hemorrhage induced by vaping. Case Rep Pulmonol. 2018;2018:9724530.

39. Khan MS, Khateeb F, Akhtar J, Khan Z, Lal A, Kholodovych V, et al. Organizing pneumonia related to electronic cigarette use: a case report and review of literature. Clinic Respir J. 2018;12(3):1295-9.

40. Flower M, Nandakumar L, Singh M, Wyld D, Windsor M, Fielding D. Respiratory bronchiolitisassociated interstitial lung disease secondary to electronic nicotine delivery system use confirmed with open lung biopsy. Respirol Case Rep. 2017;5(3):00230.

41. icpinigaitis PV, Lee Chang A, Dicpinigaitis AJ, Negassa A. Effect of e-cigarette use on cough reflex sensitivity. Chest. 2016;149(1):161-5.

42. Carson JL, Zhou L, Brighton L, Mills KH, Zhou H, Jaspers I, et al. Temporal structure/function variation in cultured differentiated human nasal epithelium associated with acute single exposure to tobacco smoke or E-cigarette vapor. Inhal Toxicol. 2017;29(3):137-44.

Cite this article as: Al-Ghamdi HS, Althagafi NG, Alshawaf MJ, Allathiqani OM, Almaflehi RA, Almuzaini FA, et al. The use of e-cigarettes and its risk of respiratory diseases. Int J Community Med Public Health 2021;8:5593-7. 